\title{
Thermal Stability of a DC/DC Converter with Inductor in Partial Saturation
}

\author{
Gianpaolo Vitale, Senior Member, IEEE, Giuseppe Lullo, Member, IEEE, \\ and Daniele Scirè, Student Member, IEEE
}

\begin{abstract}
Inductors operated in quasi saturation in DC/DC converters allow reduction of the core size and realization costs; on the other hand, they imply an increase of dissipated power that can jeopardize the thermal stability of the converter. In this paper, this issue is studied by a mathematical model able to represent both the inductor non-linearity and its temperature dependence. The main losses, such as ohmic, skin effect and magnetic, are taken into account in the model. The inductor is characterized by a polynomial curve whose parameters are a function of the temperature. Finally, the whole converter is modeled and simulation results, obtained on a boost converter, are compared with experimental measurements showing that the thermal behavior is reproduced in detail.
\end{abstract}

Index Terms- DC-DC power converters, Inductors, Nonlinear circuits, Saturation magnetization.

\section{INTRODUCTION}

$\boldsymbol{\Gamma}^{\mathrm{H}}$ HE exploitation of inductors beyond the region in which the inductance remains constant in DC/DC power converters allows attracting advantages in terms of reduction of weight and cost [1]-[4]. On the other hand, the design of a power converter becomes more complicated since there is a need for an accurate model of the inductor and the thermal stability must be verified. In fact, in a DC/DC converter, usually the inductor experiences two constant voltages during the $\mathrm{ON}$ and OFF status of the power switch. For an inductor operated in the linear zone, this produces a triangular shaped waveform. Instead, if the partial saturation (i.e. the region where the inductance swings from values close to the nominal value to values close to the saturation, known also as roll-off zone [5]) is exploited, the peak of the current is higher and losses are increased. Unfortunately, this implies a rise in the temperature producing a reduction of the inductance. It can induce a positive feedback that could increase the current until a thermal collapse occurs.

Many recently published papers deal with the issue of simulating the thermal transient concerning DC/DC switching converters.

G. Lullo, and D. Scirè, are with Dept. of Engineering University of Palermo, Palermo, Italy (e-mail: giuseppe.lullo@unipa.it, daniele.scire@unipa.it,). G. Vitale is with the Institute for high performance computing and networking (ICAR), National Research Council (CNR), Palermo, Italy (e-mail: gianpaolo.vitale@ieee.org)
Ref. [6] proposes an electrothermal model of the inductor to analyze a boost converter; it highlights that an accurate knowledge of losses in the inductor is required. The same authors explain in [7] how losses in the inductor influence the converter performance, whereas [8] proposes a 2-D model of the inductor to reduce the computational load. Ref. [9], similarly to the papers mentioned above, considers the inductor operating in a switching converter, meaning supplied by a square-wave voltage, and proposes a model of magnetic losses based on an equivalent conductance. It uses an approach based firstly on the improved Natural Steinmetz Equation $[10,11]$ and then on Generalized Steinmetz equation described in [12] and verified on several magnetic materials under different test conditions.

The operation of the inductor in the non-linear region worsens the thermal solicitation and requires proper modeling of the same inductor, as shown in [5] and further analyzed in $[13,14]$. The operation of switching power supplies with such an inductor is described in $[15,16]$.

In this paper, the inductor is modeled by a polynomial curve as in [17]. The effect of temperature has been included considering a linear dependence of the coefficients in [18], whereas in [19] the same model has been used to simulate the thermal behavior in a DC/DC converter. The improved version proposed here, including magnetic losses, allows thermal stability to be studied. The presented approach requires a low computational effort and can describe both the detail of the peak of the current when the inductor is operated in roll-off zone and the thermal behavior, including the stability.

The novelty of the paper consists in the method to analyze the thermal stability of a DC/DC converter where the inductor operates in partial saturation. It exploits a model of the inductor based on a polynomial curve taking into account the inductance versus current and temperature. This method aims to reproduce the current during operation and to study thermal stability.

The paper differs from [5,13] and from [8] since it proposes a model of the inductor based on a polynomial curve; this approach is similar to [20], but here it is improved taking into account ohmic and magnetic losses. With respect to [6], here the thermal analysis considers the non-linearity of the inductor. Finally, the analysis is explained by a mathematical model with block diagrams to make the implementation easier by a graphic interface, such as MATLAB ${ }^{\circledR}$ Simulink.

The paper is organized as follows: after this introduction in section I, focused on thermal stability issues concerning the roll-off region of the inductor, section II analyzes the losses in 
the inductor. The thermal model is presented in section III, whereas the whole model of the same inductor is discussed in section IV. The thermal stability is explained in section $\mathrm{V}$, section VI deals with the model of the DC/DC converter, section VII is devoted to the description of the experimental rig; finally, experimental results are given in section VIII.

\section{INDUCTOR LOSSES}

In this section, the losses on the inductor are analyzed concerning its behavior in a DC/DC converter. In this case, the inductor operates with a DC current bias, and it is subjected to a flux density variation due to the different values of the applied voltage depending on the state of the power switch.

A model of the losses allows calculating the amount of power that causes an increase in the temperature. The main losses considered are ohmic losses, skin effect losses, and magnetic losses.

\section{A. Ohmic losses}

Ohmic losses can be considered copper losses and depend on the wire resistance of the inductor. The wire resistance can be obtained by:

$$
R_{J}=\rho_{C u} \frac{l_{T}}{S_{C u}}
$$

where $\rho_{\mathrm{Cu}}$ is the electric resistivity of the copper wire, $\mathrm{S}_{\mathrm{Cu}}$ is its cross section area and $l_{\mathrm{T}}$ is the length of the copper conductor.

\section{B. Skin effect losses}

The high switching frequency provokes a skin effect so that the conductive current is closer to the surface of the conductors and, consequently, the resistance is increased compared to the AC value. This effect can be taken into account by the correction coefficient $K_{T}$ whose expression is given by [21]:

$$
\begin{gathered}
K_{T}=M_{T} \frac{\sinh \left(2 M_{T}\right)+\sin \left(2 M_{T}\right)}{\cosh \left(2 M_{T}\right)-\cos \left(2 M_{T}\right)}+2 M_{T} \frac{\sinh \left(2 M_{T}\right)-\sin \left(2 M_{T}\right)}{\cosh \left(2 M_{T}\right)+\cos \left(2 M_{T}\right)} \\
M_{T}=\frac{d_{\text {wire }} \sqrt{\pi}}{2 \delta_{n}}
\end{gathered}
$$

$d_{\text {wire }}$ is the copper wire diameter while $\delta_{n}$ refers to the skin depth, calculated as:

$$
\delta_{n}=\sqrt{\frac{\rho_{C u}}{\pi n f_{s} \mu_{0}}}
$$

where $n$ is the number of layers of the winding, $f_{s}$ is the switching frequency and $\mu_{\mathrm{o}}$ is the vacuum permeability. The parameter $K_{T}$ is used as correction factor of the Joule losses as shown in the block diagram of Fig. 3.

\section{Magnetic losses}

The time-average power loss per unit volume $\bar{P}_{L_{-} v}$ in an inductor core is obtained empirically by the Standard Steinmetz Equation (SSE) [8] as a function of the frequency $f$, of the flux density amplitude $B$, and of three coefficients, named $k, \alpha$ and $\beta$.

$$
\bar{P}_{L_{-} v}=k f^{\alpha} B_{\max }^{\beta}
$$

Unfortunately, the three coefficients are obtained with a sinusoidal magnetic flux density excitation by a curve fitting the losses, hence (5) is useful for loss estimation only under sinusoidal supply and cannot be employed in the considered inductor. The Generalized Steinmetz Equation (GSE) can be used to overcome this limitation [8]. In this case, the flux density and its derivative in the time domain are taken into account to calculate the instantaneous power loss $P_{L_{-} v}(t)$ :

$$
P_{L_{-} v}(t)=k_{1}\left|\frac{d B(t)}{d t}\right|^{\alpha}|B(t)|^{\beta-\alpha}
$$

The constant parameter represented by the coefficient $k_{1}$ can be evaluated by the Steinmetz parameters:

$$
k_{1}=\frac{k}{(2 \pi)^{\alpha-1}\left[\int_{0}^{2 \pi}|\cos \theta|^{\alpha}|\sin \theta|^{\beta-\alpha} d \theta\right]}
$$

The power loss per unit volume $\bar{P}_{L_{-} v}$ is achieved by a mean over the switching period $T_{s}$.

$$
\bar{P}_{L_{-} v}=\frac{1}{T_{S}} \int_{0}^{T_{S}} P_{L_{-} v}(t) \mathrm{dt}
$$

For sinusoidal flux density, (8) gives the same result as SSE (5). A more accurate evaluation is required for the waveforms typical of power converters, where the current exhibits a quasi-triangular shape with a DC offset and temperature dependency. It can be achieved by the Revised Generalized Steinmetz Equation (RGSE) [9], where the instantaneous power loss density is calculated considering only the $\mathrm{AC}$ component of the flux density as the difference between the whole flux density $B(t)$ and its mean value $B_{D C}$.

$$
B_{A C}(t)=B(t)-B_{D C}
$$

In this case, a new expression of (6) giving the power loss per unit volume in the time domain is obtained:

$$
P_{L_{-} v}(t)=k_{1}\left|\frac{d B_{A C}(t)}{d t}\right|^{\alpha}\left|B_{A C}(t)\right|^{\beta-\alpha}
$$

The total and the AC component of the instantaneous flux density are obtained as in (11) and (12) considering the voltage across the inductor $v_{L}(t)$, the number of turns $n$ and the cross-section area of the inductor $S$.

$$
B(t)=\frac{1}{n S} \int_{0}^{t} v_{L}(\tau) d \tau
$$

$B_{A C}(t)=\frac{1}{n S} \int_{0}^{t} v_{L}(\tau) d \tau-\frac{1}{T_{S}} \int_{t-T_{S}}^{t}\left(\frac{1}{n S} \int_{0}^{\tau} v_{L}\left(\tau^{\prime}\right) d \tau^{\prime}\right) d \tau$

By some manipulations of (10), considering (11) and (12), the instantaneous power loss is obtained as:

$$
P_{L}(t)=V_{e} k_{1}\left|\left(\frac{v_{L}(t)}{n S}\right)^{\alpha} B_{A C}^{\beta-\alpha}(t)\right|
$$

where $V_{e}$ is the effective volume of the core.

\section{THERMAL MODEL OF THE INDUCTOR}

As concerns the thermal model of the inductor, it has been considered as a first order system whose characteristic parameters are the thermal resistance $R_{t h}$ and the thermal capacity $C_{t h}$ [22]. These values depend on the materials, on the 
specific heat and on geometric factors. In [22] the whole thermal constant is calculated as the product of the average thermal resistance of the component towards the ambient (air) multiplied by the average thermal capacitance; it is obtained as the sum of the thermal capacitances of all of the parts of the component that, in turns, are given as the product of the specific heat values multiplied by the mass. Anyway, ref. [22] highlights that the parameters are not easy to be calculated since the heat transfer is due to conduction, convection and irradiation processes whose representing coefficients are tied to several factors such as materials, geometry, temperature and so on. By adopting the thermal model with a unique thermal constant and the experimental characterization performed by the authors, the thermal resistance of the whole inductor is extracted when the transient stabilizes based on the ratio of the generated power and the difference between the temperature of the inductor $\left(\mathrm{T}_{\text {Core }}\right)$ and environmental temperature.

Based on the power lost in the inductor, its temperature will be greater of the environmental temperature. The transfer function is the Laplace domain is given by:

$$
\frac{d \bar{P}_{L}}{d T_{\text {Core }}}=\frac{R_{t h}}{1+s \tau_{t h}}
$$

where $\tau_{t h}=R_{t h} C_{t h}$ defines the thermal constant of the inductor. The thermal resistance takes into account the heat transfer in static conditions and it is defined as the ratio of the power dissipated into the inductor $\left(\bar{P}_{L}\right)$ and the rise of its temperature $\left(\Delta T_{\text {core }}\right)$. For a better understanding of heat transfer process, the thermal quantities and equations can be associated to an electrical analogy. Based on this approach, the heat transfer rate is represented by a current generator, the temperature difference is represented by voltage, and the thermal resistances are represented by electrical resistances as shown in Fig. 1. It can be noted that the thermal constant is usually much higher than the other time constants of the converter, making the simulation difficult. In fact, to correctly reproduce the electric phenomena, a time interval considerably shorter than the switching period must be chosen. Still, since the thermal simulation requires many seconds, it implies a significant quantity of data to be managed by the simulator.

It should be remarked that in this theoretical analysis the inductor is considered at uniform temperature, following the assumption proposed in [22] and adopted by other papers dealing with the temperature of the inductor due to losses [2225]. It will be experimentally confirmed in sec. VIII.

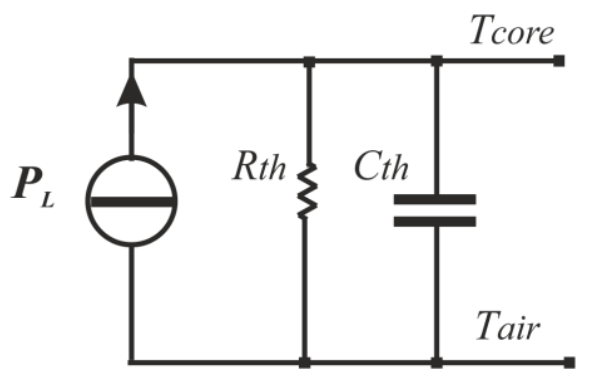

Fig. 1. Electrical lumped parameters circuit representing the heat transfer.

\section{INDUCTOR CHARACTERIZATION}

The aim of retrieving a model is to reproduce the whole electric-thermal behavior by adding two resistances based on Joule and magnetic losses respectively. The Joule effect can be easily modeled by calculating the series resistance of the copper as well as the skin losses. An equivalent resistance can be introduced to take into account magnetic losses. The inductor needs a dedicated model which considers both the non-linearity due to the operation in partial saturation region and the temperature dependence.

Finally, these models are combined to implement the inductor model which is inserted in the model of the boost converter. This model allows evaluating the stability of this process as it is explained in section $\mathrm{V}$.

\section{A. Magnetic losses modeling by equivalent resistance}

The method to model the magnetic losses by an equivalent resistance is described in [9], here only the basics are given. Differently from an ideal inductor, magnetic losses cause that an amount of the current flowing through the inductor produces heat instead of contributing to the flux density. This situation is depicted in Fig. 2, where an equivalent resistance representing magnetic losses is connected in parallel to the inductor. The ohmic resistance of the wire, taking into account also the skin effect, is connected in series since the whole current flows through it.

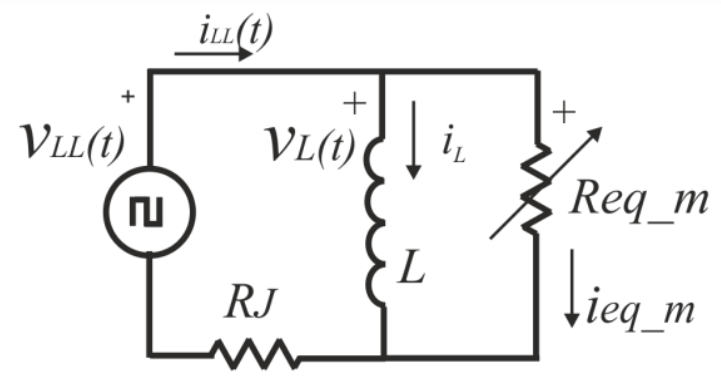

Fig. 2. Inductor equivalent circuit taking into account ohmic and magnetic losses.

As concerns the symbols: $v_{L L}(t)$ is the total voltage applied to the inductor equivalent model whereas $v_{L}(t)$ is the voltage applied to the pure inductance after subtracting the voltage drop due to Joule losses; the total current is denoted by $i_{L L}(t)$ and is given by the current $i_{L}(t)$ flowing through the inductance plus the current related to magnetic losses $i_{\text {eq_m }}(t)$.

According to [9], the equivalent resistance $R_{\text {eq }} m$ is calculated by introducing in a Type 1 feedback loop the difference between the instantaneous power loss $P_{L}(t)$ given by (13) and the instantaneous electric power $P_{E}(t)$, given by the product of the voltage and the current at the terminals of the inductor. The feedback loop will adjust the $R_{\text {eq } m}$ value in order to nullify the $\left(P_{L}-P_{E}\right)$ difference. $R_{\text {eq } \_}$will be obtained as:

$$
R_{e q_{-} m}=\frac{1}{r \int\left[P_{L}(t)-v_{L}(t) i_{L L}(t)\right] d t}
$$

where the parameter $\Upsilon \equiv\left[\mathrm{V}^{-2} \cdot \mathrm{s}^{-1}\right]$ can be adjusted to foster the speed of convergence; a unitary value has been chosen as suggested by [9]. 


\section{B. Effects of temperature}

In power inductor applications, the differential inductance defined as the ratio between the flux linkage and the current variations is lessened in the transition region [1] causing an increase of the ripple and of the power losses [26]. The effect of the temperature on the magnetic material flattens the hysteresis cycle lessening the maximum value of the flux density [27]; it implies that the slope of the hysteresis cycle, at given current, will be reduced thus experiencing a lower inductance. An increase of the temperature due to the losses will influence the current as well, creating a positive feedback loop, as can be deduced by the model of Fig. 2: the temperature will lessen the inductance $L$; hence an increase of the current $i_{L}$ is expected. Besides, the parasitic resistance $R_{J}$ will be increased with a further increase of Joule losses that depend on the square of the current. On the other hand, magnetic losses will be lessened since the increased drop voltage on $R_{J}$ reduces the voltage applied at the inductor terminals. Unfortunately, the increase of the Joule losses is higher than the lowering of the magnetic losses, and then the system is not intrinsically stable. It justifies the need to study the thermal stability.

\section{Dynamic characterization}

The most used inductors for power electronic systems are constituted by conductive material windings on a ferrite or iron powder core; the ferrite nuclei, in particular, cause a sharp and abrupt transition between the regions of low and deep saturation. In this case, the value of the inductance decreases rapidly, going towards saturation, and the current consequently rises. The behavior in partial saturation needs to be reproduced accurately, to avoid entering too deep into the saturation zone where the flux density variations are most nulled, implying high current peaks with possible damaging of the converter.

Contrarily to an inductor operated with a small current ripple, where a slight variation of the inductance value is expected so that it can be considered constant during normal operation, a broad current ripple provokes a change of the inductance during the switching period. Besides, the inductance value also depends on the temperature of the core; a higher temperature emphasizes the effect of saturation of the inductor and must be considered in the design process.

To characterize the behavior of the inductor and to manage a function computationally light that reproduces the variation of the inductance in partial saturation, a polynomial function has been chosen $[19,23]$. The coefficients of this function are considered linearly dependent on the temperature as in (16).

$$
L\left(i_{L}\right)=\sum_{m=0}^{n} L_{m}\left(1+a_{m} T_{\text {Core }}\right) i_{L}^{m}
$$

where the temperature $T_{\text {Core }}$ is in ${ }^{\circ} \mathrm{C}$ and the first index $(m=0)$ reproduces the thermal behavior when the inductance remains constant. The disadvantage of this approach consists of the necessity to identify the coefficients; regardless, for practical purposes, a third-order approximation revealed appropriated. On the other hand, once the coefficients are known, eq. (16) can be easily implemented and requires fewer calculation resources compared to the use of hyperbolic functions [5]. The polynomial curve has not been extended to model the saturation region, as performed by [5] since it is not exploited in DC/DC converters.

\section{Dynamic model including electro-thermal behavior and partial saturation}

The polynomial form identifying the inductor shown in the previous section requires the temperature as an input parameter. Temperature is cumbersome to be calculated since it varies during operation depending on some parameters such as the current that, in turn, are tied to the temperature itself. For this reason, a recursive model has been proposed in which, starting from the voltage applied to the inductor in time domain, the power loss is calculated and applied to the thermal model. It gives a new value of the temperature which allows updating the coefficients of the inductor. The block diagram is shown in Fig. 3.

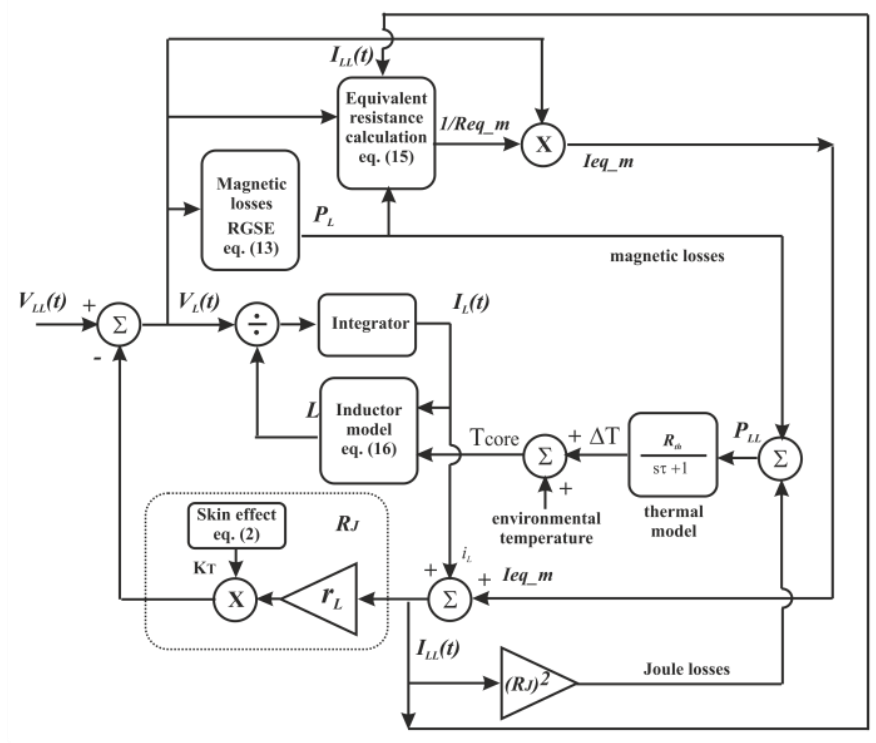

Fig. 3. Block diagram of the dynamic electro-thermal model of the inductor.

The model has the voltage of the inductor $V_{L L}(t)$ as input and retrieves its temperature and the current as output. It is focused on the model of the inductor described by eq. (16). The current through the inductor is obtained by integration of the voltage $V_{L}(t)$ obtained by $V_{L L}(t)$ subtracting the voltage drop due to the parasitic resistance of the winding and including the skin effect. The overall power losses are calculated by summing the Joule losses and the magnetic losses given by eq. (13) and used as input in the thermal model. Based on the equivalent resistance given by eq. (15), the related current $I e q \_m(t)$ is added to the current through the inductor $I_{L}(t)$ and multiplied for the parasitic resistance of the inductor $R_{J}$ to evaluate the voltage drop used to calculate $V_{L}(t)$.

\section{THERMAL StABILITY EVALUATION}

The thermal stability is evaluated by approximating the increase of the power losses, due to an increase of the temperature, as explained in sec. IV.B, with a linear relationship represented in eq. (17):

$$
\Delta P\left(T_{\text {Core }}\right)=\zeta \Delta T_{\text {Core }}
$$


In the Laplace domain, the slope $\zeta$ of the straight line in the plane $\Delta \mathrm{P}$ vs. $\Delta \mathrm{T}_{\text {Core }}$ is the transfer function giving the variation of the power for given variation of the temperature. The system, from a thermal point of view, can be represented as in Fig. 4. The open loop gain describes the increase of the temperature when an increase of power is imposed. It can be noted that for a linear inductor $\zeta \cong 0$. In this way, the stability can be analyzed as a feedback system by calculating the pole of the closed loop transfer function $F(s)$ expressing the ratio between the variations of $\mathrm{T}_{\text {Core }}$ and $\mathrm{P}$.

$$
F(s)=\frac{G(s)}{1-\zeta G(s)}=\frac{\frac{R_{t h}}{1+s \tau_{t h}}}{1-\zeta \frac{R_{t h}}{1+s \tau_{t h}}}=\frac{R_{t h}}{1-\zeta \cdot R_{t h}} \frac{1}{1+s \frac{\tau_{t h}}{1-\zeta R_{t h}}}
$$

It can be noted that the condition:

$$
\zeta \cdot R_{t h}<1
$$

defines the thermal stability of the system, as the real part of the pole in (18) will result negative. The verification of (19) depends both on the inductor and on the environment since heat sources as MOSFETs placed near the inductor, or a poor cooling, can increase $\zeta$ thus jeopardizing the stability. These are the reasons why $\zeta$ can vary from the theoretical value when it is evaluated experimentally.

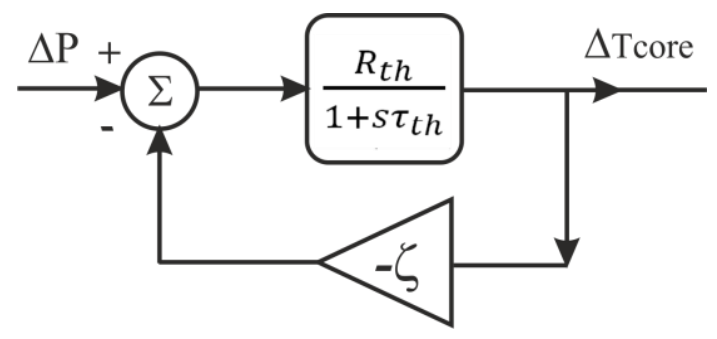

Fig. 4. Thermal equivalent representation of the converter.

\section{MOdEL OF THE BOOST CONVERTER}

The converter, modeled as in [28], has been implemented in Simulink ${ }^{\circledR}$ environment, as shown in Fig. 5a. It represents the implementation of a boost converter with the complete inductor model shown in Fig. 3, where $V_{\text {forward }}$ and $\mathrm{r}_{\mathrm{DS}}$ are the drop voltage of the free-wheel diode and the ON resistance of the MOSFET respectively, $V s$ is the supply voltage of the converter. The explanation in more detail of the block representing the parallel connection of the load is shown in Fig. 5b where the graphical representation uses only integrators (improving the numerical stability) to manage the load resistance as an external parameter to simulate a load variation. Fig. 5c shows the circuit representation of the load including the parasitic resistance (equivalent series resistanceESR) of the output capacitor $\left(r_{C}\right)$ : the current flowing through the inductor, weighted for (1-D), where D is the duty cycle of the power switch, is imposed to the load to retrieve the output voltage; it corresponds to implement the transfer function of Fig. 5d. The inductor block incorporates the dynamic model of the non-linear inductor, including the thermal behavior and the winding and magnetic losses.

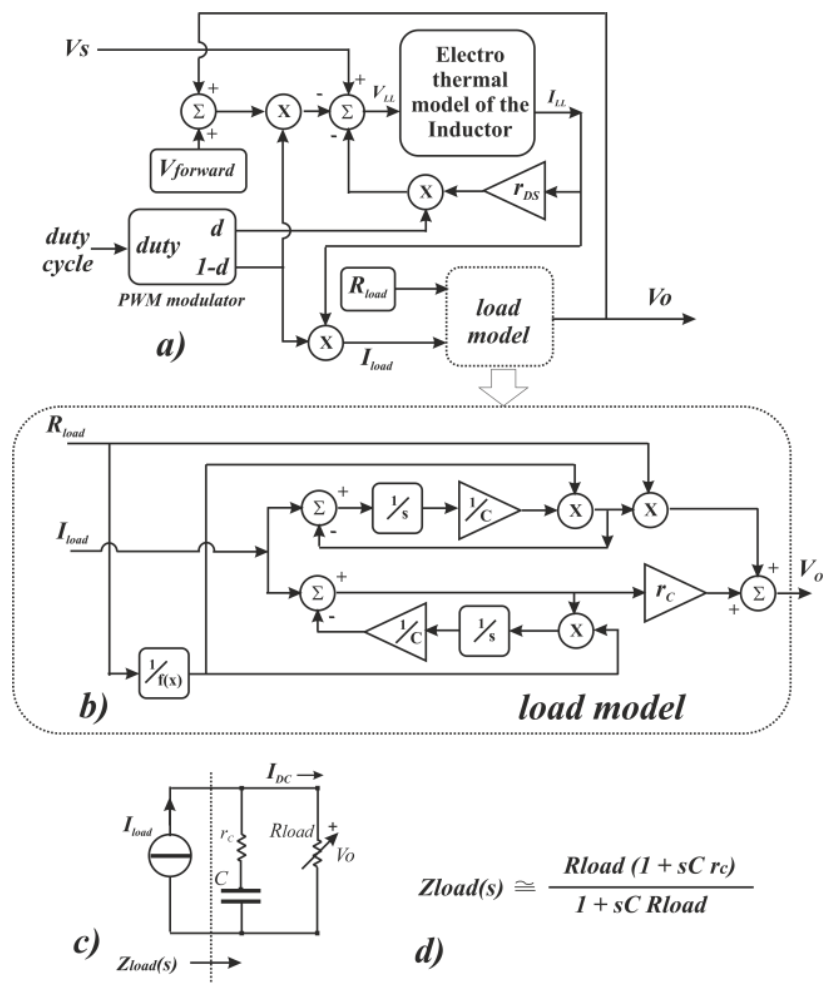

Fig. 5. a) Block diagram of the dynamic model of the converter, including the electro-thermal behavior of the inductor, b) block diagram of the load, c) equivalent circuit representation of the load, d) transfer function of the load.

\section{EXPERIMENTAL RIG}

The inductor has been characterized experimentally by a novel virtual system developed in LabVIEW $^{\circledR}$ environment [18], to identify the coefficients of (16): the system automatically performs inductance measurement for different DC current values and temperatures, providing a set of numerical values that are used to obtain the polynomial approximation. It consists of a DC/DC converter in which the inductor under test is connected. The DC current is imposed by varying the load. Different values of the ripple are obtained by varying the switching frequency.

The test has been performed on a ferrite core inductor (Panasonic model ELC18B221L), whose nominal inductance is $220 \mu \mathrm{H}$ and rated DC current is $2.4 \mathrm{~A}$. The surface $\mathrm{L}$ vs. $\mathrm{I}_{\mathrm{L}}$ vs. $\mathrm{T}_{\text {Core }}$ has been characterized up to $6 \mathrm{~A}$ and $80^{\circ} \mathrm{C}$. It has been chosen since it is a widely used inductor (CTV, VCR, Audio, PC, Fax machines, PDP, Home appliance). The experimental test has been performed considering a boost converter controlled in open loop configuration with a constant duty cycle of $50 \%$. The choice of a boost converter operating in continuous conduction mode (CCM) is justified since the inductor is subjected to high currents at its input due to the increase of the voltage at the converter output. In any case, the described approach is general; the model and method can be applied to different topologies.

The switching frequency and the load resistance of the converter have been varied during the test to obtain operating points with different DC current bias of the inductor and 
current ripple. The surface reproducing the inductance variation versus current and temperature is shown in Fig. 6. The coefficients of (16) have been obtained by interpolation, and are given in Table I. The electric signals on the inductor have been acquired by a digital oscilloscope and the temperature by a thermocouple placed on the inductor.

A relationship giving the temperature vs. the power lost on the inductor has been obtained to verify the thermal stability of the system. The power loss on the inductor is calculated by simulation for a given variation of temperature in steady-state conditions obtaining the points shown in Fig. 7. By linear regression, it is possible to retrieve the slope of the straight line described by (17), corresponding to a value of $2.7 \mathrm{~mW} /{ }^{\circ} \mathrm{C}$.

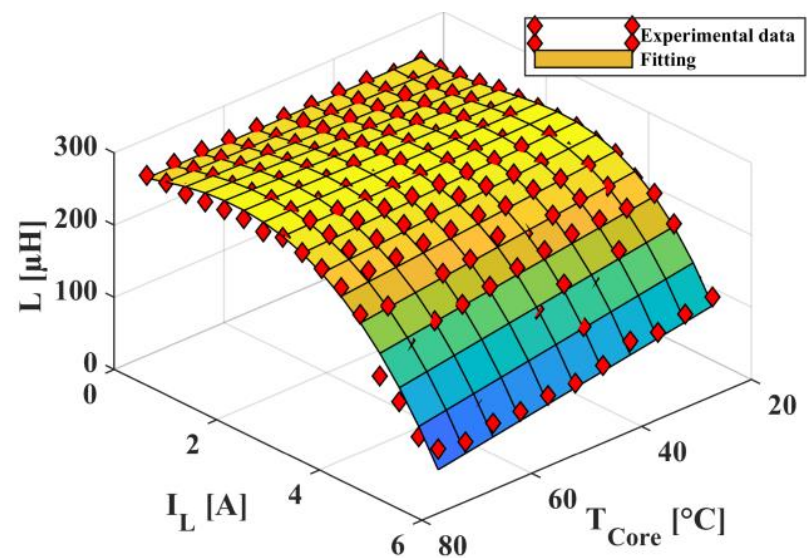

Fig. 6. Inductance variation with current and core temperature (red diamonds) and its polynomial approximation (surface).

TABLE I.

MODEL COEFFICIENTS

\begin{tabular}{ccccc}
\hline \hline Coefficient & \multicolumn{2}{c}{ Value } & Coefficient & Value \\
\hline $\mathrm{L}_{0}$ & 262 & $10^{-6}$ & $a_{0}$ & -0.0006 \\
$\mathrm{~L}_{1}$ & -28.8 & $10^{-6}$ & $a_{1}$ & -0.024 \\
$\mathrm{~L}_{2}$ & 22.9 & $10^{-6}$ & $a_{2}$ & -0.015 \\
$\mathrm{~L}_{3}$ & -3.72 & $10^{-6}$ & $a_{3}$ & -0.009 \\
\hline
\end{tabular}

Fig. 7 also shows a curve obtained considering only the first term of (16) assuming the inductor linear and varying with temperature. It can be noted that with this approximation, the slope of (17) is flat (corresponding to $\zeta=0$ ). In this case, the thermal transient is stable; however, this does not correspond to the real situation.

The values of the component of the equivalent thermal circuit have been obtained experimentally: a step variation of the current has been imposed by varying the load while the temperature has been monitored with a thermocouple.

By the evaluation of the thermal gradient, when the transient stabilizes, the thermal resistance of the whole inductor has been extracted $\left(15^{\circ} \mathrm{C} / \mathrm{W}\right)$. The thermal time constant has been measured from the evaluation of the dynamic thermal response (300 seconds). During the characterization, the temperature vs. time rises according to an exponential curve confirming the model of eq. (14). The boost converter adopts the following components: an FDP12N60NZ MOSFET for the switch, a MURB820 rectifier for the diode.
The input voltage (Vs) is $60 \mathrm{~V}$. The electric scheme is sketched in Fig. 8.

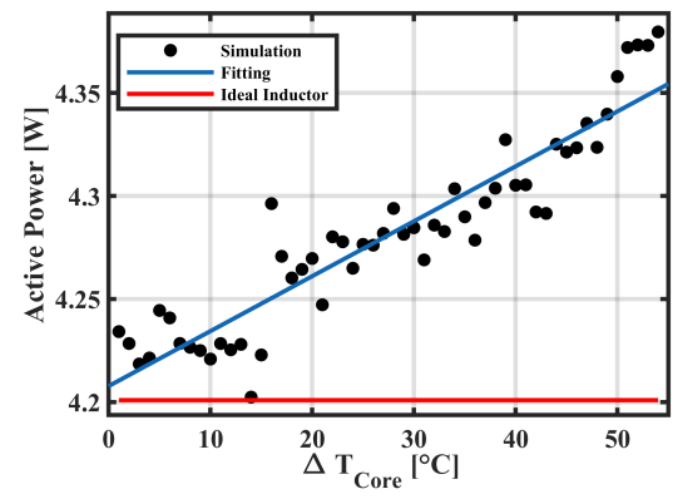

Fig. 7. Simulation of the temperature variation versus power lost on the inductor and fitting by a linear curve. The red line represents the simulation of a linear inductor when only the first term of (16) is evaluated.

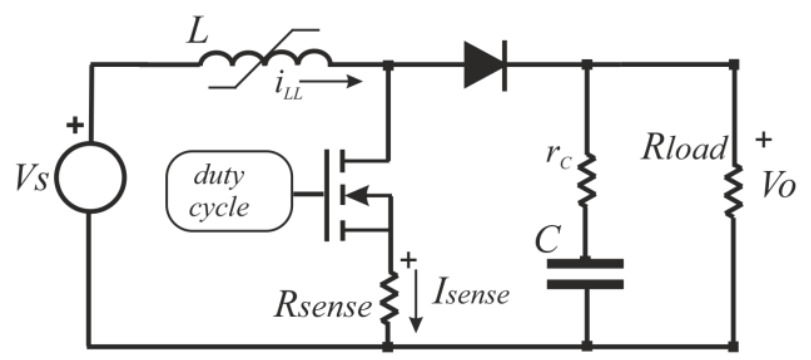

Fig. 8. Electric scheme of the converter.

\section{RESULTS}

In this section, after a thermal analysis, the experimental verification of the current flowing through the inductor by comparison with the proposed model is given. Also, the thermal stability is investigated. Three case studies are presented to validate the model. In case A), the linear operation of the inductor is reproduced, showing the current through the MOSFET ( $I_{S E N S E}$ ) with two different values of the load current $\left(I_{D C}\right)$ obtained by varying the load resistance of the converter. The current ripple does not vary, as expected. The maximum value of the current assures the operation in the linear zone. In case B), the current ripple is increased by reducing the switching frequency to $50 \mathrm{kHz}$; again, two different $\mathrm{I}_{\mathrm{DC}}$ values are considered. It can be noted that the higher ripple increases losses and, consequently, the temperature. In case C), the ripple is further increased, and the operation exploits the quasi-saturation zone showing a different shape of waveforms depending also on the temperature of the inductor that further increases due to the operation in partial saturation. Finally, the thermal collapse is described.

\section{A. Thermal analysis}

This analysis aims at verifying that during operation: a) the temperature of the core is influenced neither by the heatsink of the MOSFET nor by the thermocouple, b) the temperature of the core can be considered uniform. The distribution of the 
temperature has been measured by a thermal imaging camera model FLIR SC660; its temperature measurement capability ranges from $-40{ }^{\circ} \mathrm{C}$ to $1500{ }^{\circ} \mathrm{C}$ with $1{ }^{\circ} \mathrm{C}$ accuracy.

A prototype of the circuit is shown in Fig. 9, where it can be noted on the left side that a thermal insulation sheet has been glued on one side of the heatsink; the right side shows a detail of the thermocouple to measure the temperature of the inductor; a small quantity of thermal paste is used to improve the contact. The mass of the thermocouple is negligible compared to the inductor. Fig. 10 shows the thermal image of the circuit. It can be observed that the temperature exhibits a variation of few $\%$ on the top side of the ferrite. Besides, the thermal sheet avoids heat radiation from the heatsink towards the inductor, and the MOSFET remains colder than the inductor. Finally, the temperature of the copper is slightly higher on the top side whereas it diminishes near the bottom. It has been verified in all performed tests.

As an example of temperature distribution, during a test, the following values have been acquired: in the center of the top side $\mathrm{T}_{\text {top }}=81^{\circ} \mathrm{C}$; in the outer circumference of the top side $\mathrm{T}_{\mathrm{ext}}=79^{\circ} \mathrm{C}$; on the external surface of copper $\mathrm{T}_{\mathrm{Cu} \text { top }}=83^{\circ} \mathrm{C}$ near the top and $\mathrm{T}_{\mathrm{Cu} \text { bottom }}=80^{\circ} \mathrm{C}$ close the bottom. The MOSFET case temperature was equal to $71^{\circ} \mathrm{C}$.

As concerns the following tests, the indicated temperature refers to the one measured on the center of the top.
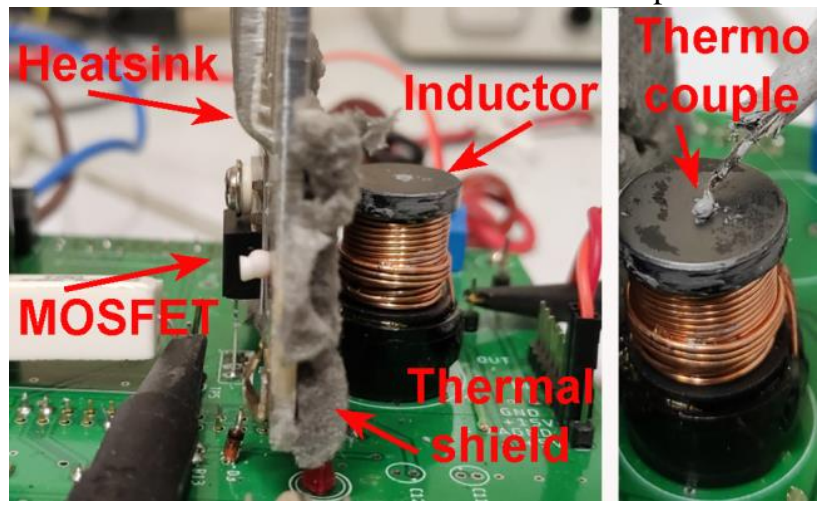

Fig. 9. Left: picture of the circuit with the main elements; right: detail of the inductor with the thermocouple placed at the center of the top side.

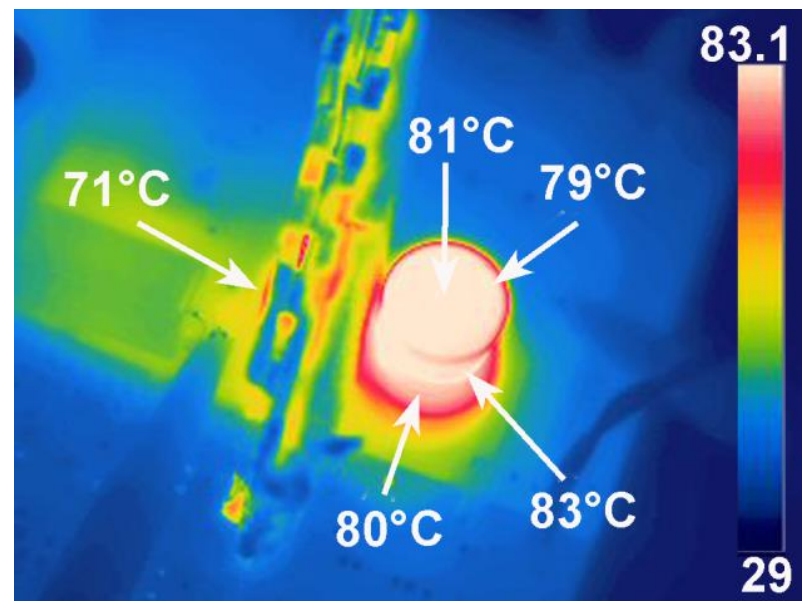

Fig. 10. Thermal image of the inductor with the temperatures measured in some relevant points.
During measurements, the environment temperature was $\mathrm{T}_{\mathrm{env}}=25^{\circ} \mathrm{C}$ with a relative humidity of $66 \%$.

\section{B. Case study 1}

Fig. 11 shows the comparison between the simulation and experimental waveforms of the current $\mathrm{I}_{\mathrm{SENSE}}$, maintaining constant the switching frequency $F_{s}=100 \mathrm{kHz}$ with $\mathrm{I}_{\mathrm{DC}} \approx 2 \mathrm{~A}$ and $\mathrm{T}_{\text {Core }}=45^{\circ} \mathrm{C}$ in comparison with the one at $\mathrm{I}_{\mathrm{DC}} \approx 4 \mathrm{~A}$ and $\mathrm{T}_{\text {Core }}=45^{\circ} \mathrm{C}$. In Table II, the comparison between the simulated and experimental output voltages (mean value \pm one half of the ripple) is shown. It can be noted that in these two conditions, the inductor operates in the linear region and the inductance remains constant as well as the current ripple, whose peak value is about $1 \mathrm{~A}$.

TABLE II.

OUTPUT VOLTAGE FOR $\mathrm{T}_{\mathrm{CORE}}=45^{\circ} \mathrm{C}, \mathrm{F}_{\mathrm{S}}=100 \mathrm{KHz}$

\begin{tabular}{ccc}
\hline \hline I $_{\text {DC }}$ [A] & V $_{\text {OUT EXP. [V] }}$ & V $_{\text {OUT SIM. [V] }}$ \\
\hline 2.0 & $118.7 \pm 1.1$ & $118.4 \pm 0.8$ \\
4.0 & $118.7 \pm 1.2$ & $118.4 \pm 0.8$ \\
\hline
\end{tabular}

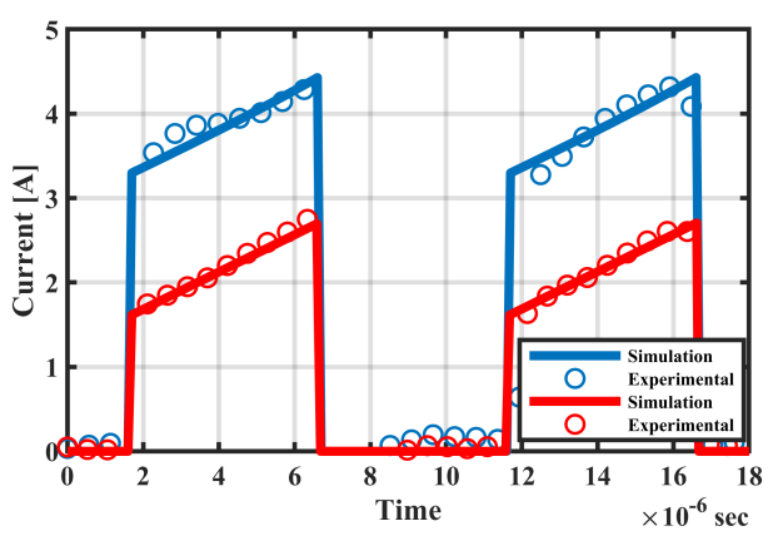

Fig. 11. Comparison between simulation and experimental results of the output current waveforms maintaining constant the frequency, $\mathrm{F}_{\mathrm{S}}=100 \mathrm{kHz}$, with (red) $\mathrm{I}_{\mathrm{DC}} \approx 2 \mathrm{~A}, \mathrm{~T}_{\text {Core }}=45^{\circ} \mathrm{C}$, and (blue) $\mathrm{I}_{\mathrm{DC}} \approx 4 \mathrm{~A}, \mathrm{~T}_{\text {Core }}=45^{\circ} \mathrm{C}$.

\section{Case study 2}

In this case, the switching frequency is lowered to $50 \mathrm{kHz}$. Fig. 12 shows the current $I_{\text {SENSE }}$ obtained for $\mathrm{I}_{\mathrm{DC}} \approx 3 \mathrm{~A}$ and $\mathrm{T}_{\text {Core }}=55^{\circ} \mathrm{C}$; the same figure shows the same current obtained for $\mathrm{I}_{\mathrm{DC}} \approx 4 \mathrm{~A}$ and $\mathrm{T}_{\text {Core }}=55^{\circ} \mathrm{C}$. In this operating condition, the current ripple is increased, reaching a peak value of $2 \mathrm{~A}$, and the operating point is placed between the linear region and the roll-off region; the current still raises as a straight line in the first case, with a slight upward bending in the second case.

Even if the maximum current on the inductor is near the rated value, the inductance does not decrease since the temperature remains relatively low; moreover, the core temperature is stabilized showing that the operation in linear zone does not provoke a positive thermal feedback. From the output voltages (mean value \pm one half of the ripple) listed in Table III, it is possible to note a slight decrease compared to the values of Table II. It is caused by the increase of the voltage drops on the parasitic resistances of the converter. In any case, experimental results agree with the values obtained by the model. 


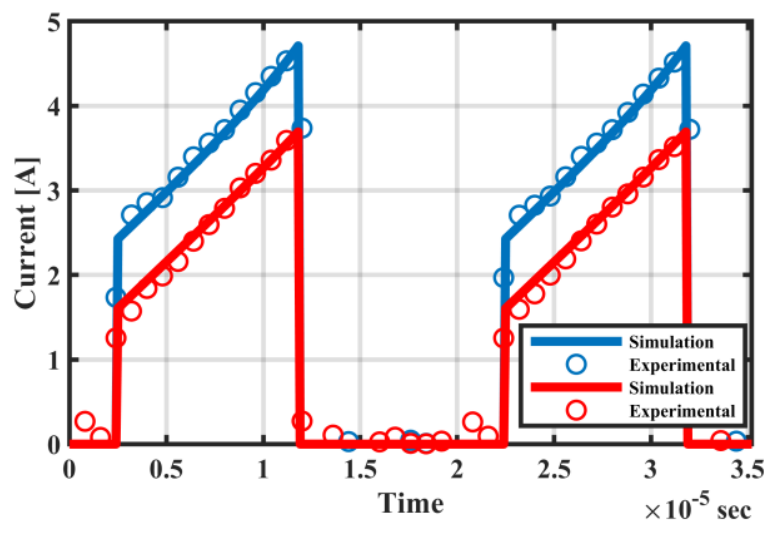

Fig. 12. Comparison between simulation and experimental results of the output current waveforms maintaining constant the frequency, $\mathrm{Fs}=50 \mathrm{kHz}$, with (red) $\mathrm{I}_{\mathrm{DC}} \approx 3 \mathrm{~A}, \mathrm{~T}_{\text {Core }}=55^{\circ} \mathrm{C}$, and (blue) $\mathrm{I}_{\mathrm{DC}} \approx 4 \mathrm{~A}, \mathrm{~T}_{\text {Core }}=55^{\circ} \mathrm{C}$.

TABLE III.

OUTPUT VOLTAGE FOR $\mathrm{T}_{\mathrm{CORE}}=55^{\circ} \mathrm{C}, \mathrm{F}_{\mathrm{S}}=50 \mathrm{KHz}$

\begin{tabular}{ccc}
\hline \hline $\mathbf{I}_{\text {DC }}[\mathbf{A}]$ & $\mathbf{V}_{\text {OUT EXP. }}$ [V] & $\mathbf{V}_{\text {OUT SIM. }}[\mathbf{V}]$ \\
\hline 3.0 & $114.7 \pm 2.1$ & $115.1 \pm 1.7$ \\
4.0 & $112.8 \pm 2.2$ & $112.7 \pm 1.9$ \\
\hline
\end{tabular}

\section{Case study 3}

Fig. 13 shows the comparison of simulation and experimental values of the current $I_{\text {SENSE }}$ obtained with a switching frequency of $30 \mathrm{kHz}$ and $\mathrm{I}_{\mathrm{DC}} \approx 3 \mathrm{~A}$. In this case, the increase of the current ripple provokes the non-linearity of the inductor. In particular, the load current is given for different temperatures of the inductor: $50^{\circ} \mathrm{C}$ (Fig. 12a), $75^{\circ} \mathrm{C}$ (Fig. 12b), $85^{\circ} \mathrm{C}$ (Fig. 12c). It can be noted that the peak of the current becomes evident as the temperature is raised.

For the sake of clarity, a detail of the maximum current is shown in Fig. 14 where the currents obtained for $\mathrm{T}_{\text {Core }}=50^{\circ} \mathrm{C}$, $75^{\circ} \mathrm{C}$, and $85^{\circ} \mathrm{C}$, are displayed. It can be appreciated a rise of the current peak from $5.3 \mathrm{~A}$ to $6.1 \mathrm{~A}$ obtained at $85^{\circ} \mathrm{C}$.

In order to obtain useful information about the maximum rated current, simulations for case 3 have been repeated including different temperatures. The resulting maximum current peak and ripple values are given in Table IV. It can be noted that, as the temperature rises, the system experiences an increase in the peak as well as an extension of the ripple. Moreover, the mean output voltage diminishes due to the increase in the peak of the current through the MOSFET. In such circumstances, if the converter is driven by a voltage controller, it will try to remedy this by increasing the duty cycle, causing a further increase of the current peak. For this reason, a more advanced control strategy shall be adopted, also involving a feedback loop for the current.

Finally, the thermal transient has been calculated; the growth of the temperature has been reproduced by the model shown in Fig. 4 with a simulated time of about 600s. The thermal transient of the inductor is represented in Fig. 15: the inductor stabilizes its temperature at a value of about $90^{\circ} \mathrm{C}$, which is reflected well in the experimental data. In this case, the slope of the power regression (eq. 17) corresponds to a value of $2.7 \mathrm{~mW} /{ }^{\circ} \mathrm{C}$. It can be remarked that, during transient, the peak of the current flowing through the inductor (and consequently through the MOSFET) rises of about $20 \%$.
The system shows a stable transient for values of the $\zeta$ up to $67 \mathrm{~mW} /{ }^{\circ} \mathrm{C}$ coherently with the stability condition (19). For the sake of clarity, the same figure shows a different unstable case in which the $\zeta$ is raised to $100 \mathrm{~mW} /{ }^{\circ} \mathrm{C}$. Such value is higher than the stability threshold defined in Eq. (19), and the temperature, therefore, dramatically grows.

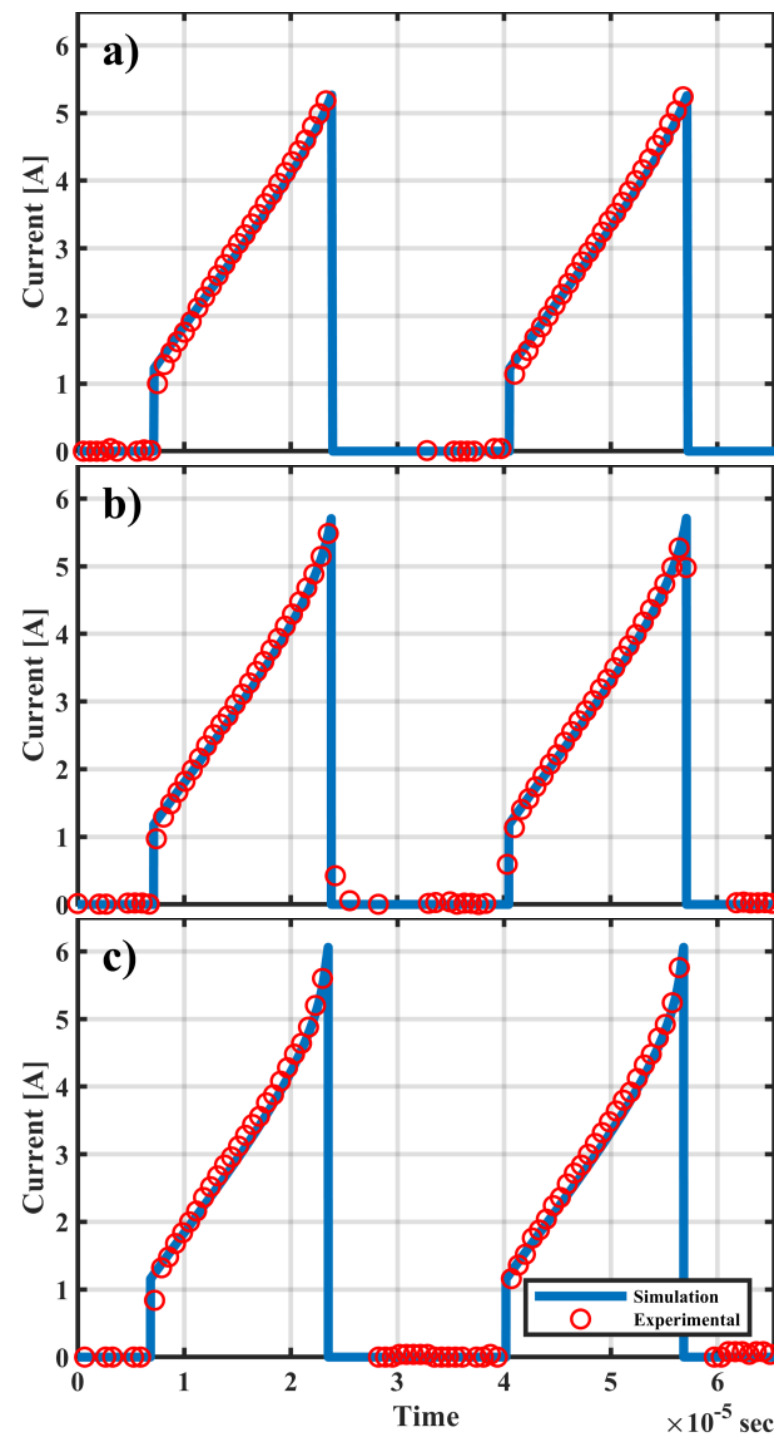

Fig. 13. Comparison between simulation and experimental results of the current waveforms maintaining constant the load current $\left(\mathrm{I}_{\mathrm{DC}} \approx 3 \mathrm{~A}\right)$ and the switching frequency $\left(F_{s}=30 \mathrm{kHz}\right)$ for different temperatures of the core: a) $\left.\left.\mathrm{T}_{\text {Core }}=50^{\circ} \mathrm{C}, \mathrm{b}\right) \mathrm{T}_{\text {Core }}=75^{\circ} \mathrm{C}, \mathrm{c}\right) \mathrm{T}_{\text {Core }}=85^{\circ} \mathrm{C}$.

In such a condition, a severe failure of the converter can occur; particularly, the heat generated by the inductor leads to a rise in the internal temperature, exceeding the operating temperature reported in the datasheet and, ultimately, causing a destructive collapse of the component.

Specifically, the windings are insulated with polymers such as polyurethane, polyamide and polyester, which cannot generally exceed the temperature of $80-150 \quad{ }^{\circ} \mathrm{C}$ [23]. Exceeding this limit causes the degradation of the insulating film covering the copper, implying short circuits among wires. It should be remarked that this fault occurs at a much lower temperature than the Curie point, jeopardizing an intrinsic 
advantage of the ferrite, and dramatically contributes to the failure of the inductor. An example of a damaged inductor is shown in Fig. 16.

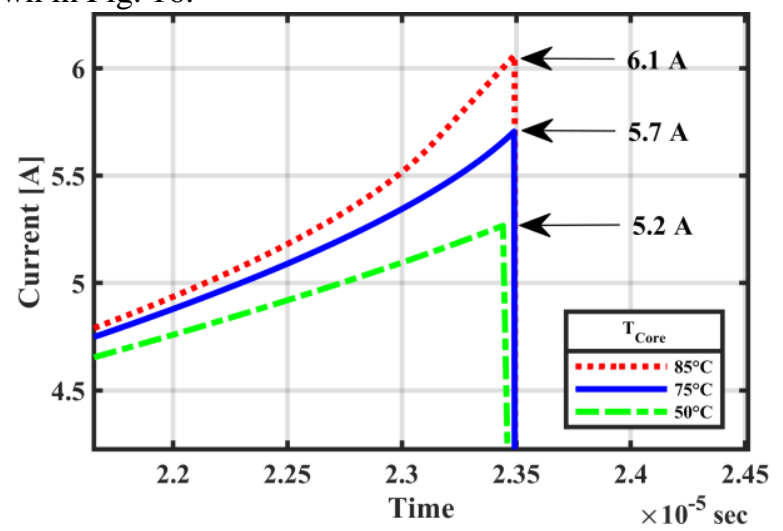

Fig. 14. Comparison of the peak of the current in the inductor maintaining constant the load current $\left(\mathrm{I}_{\mathrm{DC}} \approx 3 \mathrm{~A}\right)$ and the switching frequncy $\left(F_{s}=30\right.$ $\mathrm{kHz}$ ) for different temperatures of the core .

TABLE IV.

CurRent PEAK AND RIPPle VALUES For DifFERENT TEMPERATURES

\begin{tabular}{ccc}
\hline \hline Temperature $\left[{ }^{\circ} \mathbf{C}\right]$ & Ripple $[\mathbf{A}]$ & Peak $[\mathbf{A}]$ \\
\hline 25 & 3.78 & 5.01 \\
50 & 4.02 & 5.23 \\
60 & 4.18 & 5.38 \\
75 & 4.49 & 5.66 \\
85 & 4.86 & 6.06 \\
\hline
\end{tabular}

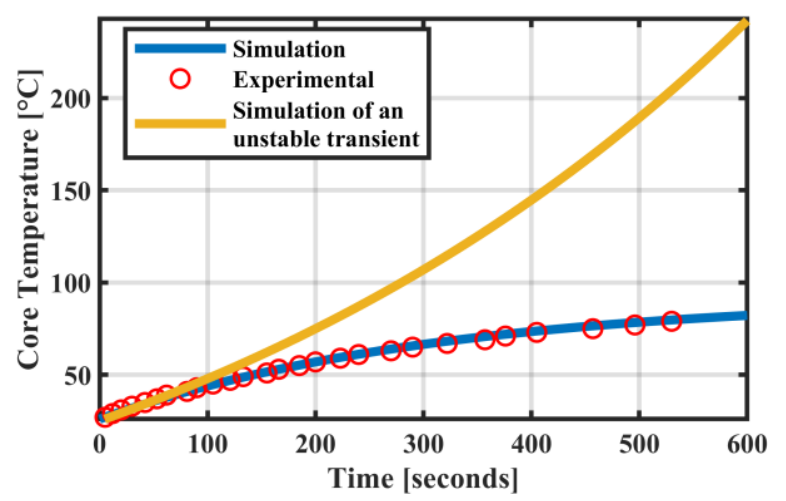

Fig. 15. Comparison among experimental thermal transient (red circles) and the simulated one (blue line) with $F_{s}=30 \mathrm{kHz}, \mathrm{I}_{\mathrm{DC}} \approx 3 \mathrm{~A}$. The orange line represents a simulation of a thermal transient with the same operating condition but with a $\zeta$ of $100 \mathrm{~mW} /{ }^{\circ} \mathrm{C}$.

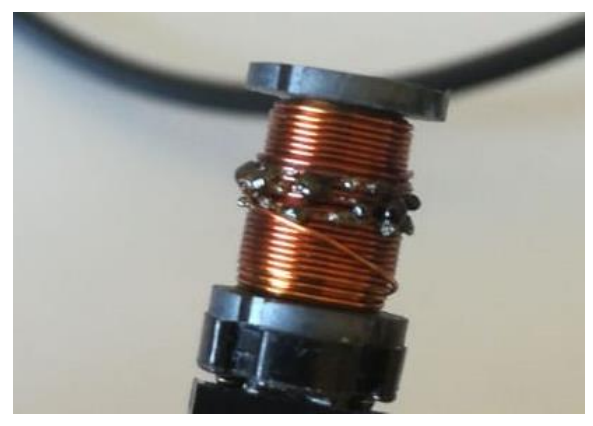

Fig. 16. Photograph of an inductor damaged by thermal collapse.

\section{CONCLUSION}

A mathematical model for inductors operated in partial saturation, taking into account the temperature, is retrieved. The model exploits a polynomial curve and is conceived for DC/DC converters. A method is devised allowing the analysis of thermal transients during the operation of the converter; it includes the variation of the inductance versus the temperature. The proposed approach avoids a significant number of data to be managed differently from the classical transient analysis time-consuming methods due to significant differences in thermal time constants and the period of the control signal.

As an example, the thermal stability of a boost converter is analyzed. In this way, it is possible to reproduce the current flowing through the inductance, showing the characteristic shape representing the operation in partial saturation.

The proposed thermal characterization approach is based on a first-order thermal model identified by measurements; it gives a representation in operating conditions of temperature influencing the behavior of the inductance.

Experimental results confirm the accordance between the current obtained by simulation and the measured values. The linear behavior can be obtained as a particular case. The method for characterizing the inductor is general, as well as its application in different DC/DC converters.

A stability condition is discussed. By showing thermal transients, it is also explained that thermal stability is tied both to the inductor and to the converter layout and that thermal collapse can occur under certain conditions jeopardizing the inductor reliability and, consequently, the whole reliability of the converter.

\section{ACKNOWLEDGMENT}

The authors are grateful to Prof. Antonino Maltese and Prof. Giuseppe Ciraolo from the Department of Engineering, University of Palermo, for performing thermal measurements and for image processing.

\section{REFERENCES}

[1] M. S. Perdigão, J. P. F. Trovão, J. M. Alonso, and E. S. Saraiva, "LargeSignal Characterization of Power Inductors in EV Bidirectional DC-DC Converters Focused on Core Size Optimization," IEEE Trans. Ind. Electron., vol. 62, no. 5, pp. 3042-3051, 2015.

[2] D. K. Saini, A. Ayachit, A. Reatti, and M. K. Kazimierczuk, "Analysis and Design of Choke Inductors for Switched-Mode Power Inverters," IEEE Trans. Ind. Electron., vol. 65, no. 3, pp. 2234-2244, Mar. 2018.

[3] Z. Ozkan and A. M. Hava, "Current Control of Single-Phase VSC Systems With Inductor Saturation Using Inverse Dynamic Model-Based Compensation," IEEE Trans. Ind. Electron., vol. 66, no. 12, pp. 92689277, Dec. 2019.

[4] J. M. Schellekens, J. L. Duarte, H. Huisman, and M. A. M. Hendrix, "Volume reduction of opposed current converters through coupling of inductors and interleaved switching," in IECON Proc. (Industrial Electronics Conference), 2012, pp. 852-857.

[5] G. Di Capua and N. Femia, "A novel method to predict the real operation of ferrite inductors with moderate saturation in switching power supply applications," IEEE Trans. Power Electron., vol. 31, no. 3, pp. 2456-2464, 2016.

[6] K. Górecki and K. Detka, "Application of Average Electrothermal Models in the SPICE-Aided Analysis of Boost Converters," IEEE Trans. Ind. Electron., vol. 66, no. 4, pp. 2746-2755, 2019. 
[7] K. Gorecki and K. Detka, "Influence of Power Losses in the Inductor Core on Characteristics of Selected DC-DC Converters," Energies, vol. 12, no. 10, p. 1991, 2019.

[8] R. A. Salas and J. Pleite, "Equivalent electrical model of a ferrite core inductor excited by a square waveform including saturation and power losses for circuit simulation," IEEE Trans. Magn., vol. 49, no. 7, pp. 4257-4260, 2013.

[9] A. Abramovitz and S. S. Ben-Yaakov, "RGSE-Based SPICE Model of Ferrite Core Losses," IEEE Trans. Power Electron., vol. 33, no. 4, pp. 2825-2831, 2018.

[10] A. Van Den Bossche, V. C. Valchev, and G. B. Georgiev, "Measurement and loss model of ferrites with non-sinusoidal waveforms," in PESC Record - IEEE Annual Power Electronics Specialists Conference, 2004, vol. 6, no. 1, pp. 4814-4818.

[11] V. C. Valchev, A. P. Van Den Bossche, and D. M. Van De Sype, "Ferrite losses of cores with square wave voltage and DC bias," IECON Proc. (Industrial Electron. Conf., vol. 2005, pp. 837-841, 2005.

[12] J. Mühlethaler, J. Biela, J. W. Kolar, and A. Ecklebe, "Core losses under the DC bias condition based on steinmetz parameters," IEEE Trans. Power Electron., vol. 27, no. 2, pp. 953-963, 2012.

[13] K. Stoyka, G. Di Capua, and N. Femia, "A Novel AC Power Loss Model for Ferrite Power Inductors," IEEE Trans. Power Electron., vol. 34, no. 3, pp. 2680-2692, 2019.

[14] G. Di Capua, N. Femia, and K. Stoyka, "A generalized numerical method for ferrite inductors analysis in high current ripple operation," Integr. VLSI J., vol. 58, no. Jan., pp. 473-484, 2017.

[15] G. Di Capua, N. Femia, and K. Stoyka, "Switching Power Supplies with Ferrite Inductors in Sustainable Saturation Operation," Int. J. Electr. Power Energy Syst., vol. 93, pp. 494-505, 2017.

[16] Q. Li, D. Jiang, and Y. Zhang, "Analysis and Calculation of Current Ripple Considering Inductance Saturation and Its Application to Variable Switching Frequency PWM," IEEE Trans. Power Electron., pp. $1-1,2019$.

[17] G. Lullo, D. Scirè, and G. Vitale, "Non-linear inductor modelling for a DC/DC Buck converter," Renew. Energy Power Qual. J., vol. 1, no. 15, pp. 686-693, 2017.

[18] D. Scirè, S. Rosato, G. Lullo, and G. Vitale, "Characterization of Nonlinear Inductors Including Thermal Effects for Power Applications," Renew. Energy Power Qual. J., vol. 1, no. April, pp. 728-734, 2018.

[19] D. Scire, S. Rosato, G. Lullo, G. Vitale, "A Temperature Dependent Non-Linear Inductor Model for a DC/DC Boost Converter," in SMACD $15^{\text {th }}$ conference, Sept. 2018, pp. 237-240.

[20] S. F. Roberto, D. Scire, G. Lullo, G. Vitale, "Equivalent Circuit Modelling of Ferrite Inductors Losses," in IEEE 4th International Forum on Research and Technologies for Society and Industry, RTSI Proc. 2018.

[21] S. De Simone, C. Adragna, C. Spini, and G. Gattavari, "Design-oriented steady state analysis of LLC resonant converters based on FHA," in International Symposium on Power Electronics, Electrical Drives, Automation and Motion,. SPEEDAM 2006, vol. 2006, pp. 200-207.

[22] Valchev, V. C., \& Van den Bossche, A. (2018). Inductors and transformers for power electronics. CRC Press, Taylor \& Francis Group, Boca Raton, FL (2005).

[23] A. Barili, et. Al., "A Simulation Model for the Saturable Reactor," IEEE Trans. Ind. Electron., vol. 35, no. 2, pp. 301-306, 1988.

[24] Valchev, V. C., \& Van den Bossche, A. (2018). Inductors and transformers for power electronics. CRC Press, Taylor \& Francis Group, Boca Raton, FL (2005).

[25] M. Sippola, and R. E. Sepponen, "Accurate prediction of highfrequency power-transformer losses and temperature rise", IEEE Tran. Power Electron., vol. 17, no. 5, pp. 835-847, (2002).

[26] J. Muhlethale , et. Al, "Improved core-loss calculation for magnetic components employed in power electronic systems", IEEE Trans. on Power electron. vol 27, no 2, pp. 964-973. (2011).

[27] M. S. Rylko, J. G. Hayes, and M. G. Egan, "Experimental investigation of high-flux density magnetic materials for high-current inductors in hybridelectric vehicle DC-DC converters,". IEEE VPPC Proc., 2010.

[28] C. W. T. McLyman, Transformer and inductor design handbook. CRC press, Taylor \& Francis Group. fourth ed., Boca Raton, FL, 2011.
[29] G. Vitale, "Analysis of DC/DC converters by mean node voltage method", Renewable Energy and Power Quality Journal (RE\&PQJ) ISSN 2172-038 X, No.13, April 2015.

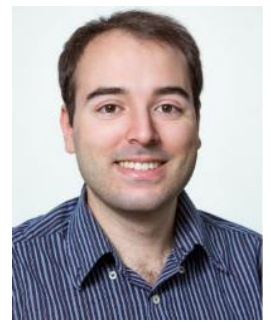

D. Scirè (S'20) was born in Palermo, Italy, in 1991. He received the received the B.S. and M.S. degrees in electronic engineering from University of Palermo, Palermo, Italy, in 2014 and 2017, respectively.

$\mathrm{He}$ is currently working towards the Ph.D. degree in Information and Communication Technologies at University of Palermo, Palermo, Italy. From 2018 to 2019 , he was a visiting $\mathrm{PhD}$ student with the Photovoltaic Material and Devices group, Delft University of Technology, Delft, the Netherlands. His research interests include modelling and design of solar cells, power converters and magnetic components. Mr. Scirè was a recipient of the European Material Research Society Graduate Student Award in 2019.

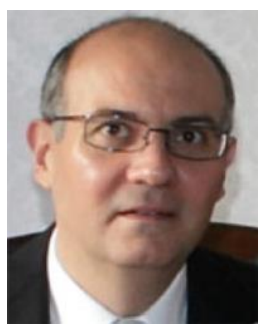

G. Lullo (M'00) was born in Palermo, Italy, in 1965. He received the M.S. degree with honors and the Ph.D. degree in electronic engineering from the University of Palermo, Palermo, Italy, in 1990 and 1995, respectively. He was a Visiting Research Fellow (1993 - 1994) within the Optoelectronics Laboratory, Glasgow University, U.K., and a Visiting Scientist (1998) within the Department of Electronics and Computer Science, Massachusetts Institute of Technology, Cambridge, MA, USA. In 1999 he became a Researcher at the University of Palermo and in 2005 an Associate Professor of electronics at the same university. During the years he has taught several courses on electronic devices, electronics basics, electronics for telecommunications, analog electronics, and applied electronics.

$\mathrm{He}$ is currently in charge for the Applied Electronics Laboratory at the University of Palermo. His research activities have covered several areas, regarding the design and fabrication of optoelectronic and electronic devices, the design of custom optical systems, the design of mixed-signal electronic systems, and electronics for controlling X-ray satellite telescopes. More recently he has been involved in research in the field of power electronic systems. He holds a patent on an optical diffractometer. He also founded and co-managed for twenty years, as a Chief Technical Officer, Microtech s.r.l., a high-tech spin-off company devoted to the realization of laser direct writing equipments for microlithographic processes. Dr. Lullo is a member of IEEE and of the Italian National Institute for Astrophysics.

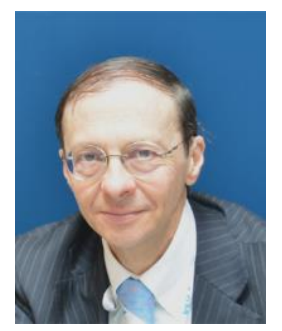

G. Vitale (M'04-SM'13) was born in Palermo, Italy, in 1964. He received the M.S. degree with honors in electronic engineering from University of Palermo, Palermo, Italy, in 1988.

$\mathrm{He}$ is senior researcher of the National Research Council of Italy (CNR) since 1994. $\mathrm{He}$ works at the Institute for high performance computing and networking (ICAR). He received the national scientific qualification ("Abilitazione scientifica nazionale") as full professor on electric energy engineering (cod. 09/E2) in 2013 and on electronic energy engineering (cod. 09/E3) in 2017. He taught "power electronics" and "applied electronics", now he teaches "Industrial electronics" at the University of Palermo, Department of Engineering, MD electronics engineering.

Prof. G. Vitale is the co-author of two books, two edited books, four book chapters and over one hundred scientific articles, of which 40 were published on international ISI journals. He holds a US patent $(9,856,857)$ on a method for converting energy from a wind tubine. He is senior member of IEEE (Institute of Electrical and Electronics Engineers) and serves as reviewer for several journals and conferences. His current research interests are in the fields of Power Electronics, Power Generation from renewable sources, Electromagnetic Compatibility and Robotics. 